\title{
GENETIC ASSOCIATION OF SOLUTE CARRIER TRANSPORTER GENE VARIANTS WITH METFORMIN RESPONSE
}

\author{
Abrahams-October $Z^{1}$, Xhakaza L ${ }^{1}$, Pearce $\mathrm{B}^{1, *}$, Mandisa Masilela $\mathrm{C}^{1}$, \\ Benjeddou $\mathrm{M}^{1}$, Vincent Adeniyi $\mathrm{O}^{2}$, Johnson $\mathrm{R}^{3,4}$, Jebio Ongole $\mathrm{J}^{5}$ \\ *Corresponding Author: Brendon Pearce, Ph.D., Department of Biotechnology, University of the \\ Western Cape, Private Bag X17, Bellville 7535, South Africa. Tel.: +2721-959-2080. Fax: +2721-959- \\ 2648. E-mail: brendon.biff@gmail.com
}

\begin{abstract}
Type 2 diabetes mellitus (T2DM) is a metabolic disorder characterized by elevated blood glucose levels and is influenced by both genetic and environmental factors. It is treated with various classes of oral antidiabetic drugs, however, response to treatment is highly variable with patients failing to achieve adequate glycemic control. Treatment response variability has been associated with single nucleotide polymorphisms (SNPs) which influence the pharma-cokinetics and pharmacodynamics of $\operatorname{drug}(\mathrm{s})$. The aim of this study was to evaluate the genetic association of 17 SNPs and the response to metformin therapy in patients diagnosed with diabetes from the indigenous Nguni population of South Africa. One hundred and forty indigenous African patients diagnosed with T2DM were recruited and genotyped using the MassARRAY ${ }^{\circledR}$ system. Therapeutic response of patients was ascertained by a change in $\mathrm{Hb} \mathrm{A}_{1 \mathrm{c}}$. Two SNPs (rs1801282 and rs6265) were monomorphic. All other variants were within the HardyWeinberg equilibrium (HWE). The T allele of the SLC variant rs 316009 [odds ratio $(\mathrm{OR})=0.25,95 \%$ confidence interval $(95 \% \mathrm{CI})=0.01-0.09, p$ value $=0.044]$ and the CT genotype of the $P C K 1$ variant $\mathrm{rs} 4810083(\mathrm{OR}=2.80$,
\end{abstract}

\footnotetext{
${ }^{1}$ Precision Medicine Unit, Department of Biotechnology, Faculty of Natural Sciences, University of the Western Cape, Bellville, South Africa

${ }^{2}$ Department of Family Medicine, Walter Sisulu University, East London, South Africa

${ }^{3}$ South African Medical Research Council, Parow, Cape Town, South Africa

${ }^{4}$ Division of Medical Physiology, Faculty of Medicine and Health Sciences, Stellenbosch University, Tygerberg, South Africa

${ }^{5}$ Department of Family Medicine, Piet Retief Hospital, Mkhondo, Mpumalanga, South Africa
}

$95 \% \mathrm{CI}=1.01-7.79, p$ value $=0.049)$ were associated with an improved response to treatment after adjustment. No association was observed with post Bonferroni correction. Moreover, this study provides important additional data regarding possible associations between genetic variants and metformin therapy outcomes. In addition, this is one of the first studies providing genetic data from the understudied indigenous sub-Saharan African populations.

Keywords: Single nucleotide polymorphisms (SNPs); SLC variants; South African cohort; Treatment response; Type 2 diabetes mellitus (T2DM).

\section{INTRODUCTION}

The prevalence of diabetes mellitus (DM) across the world is constantly rising. It is estimated that 642 million cases of DM will be reported by the year 2040 [1]. In the African region alone, it was found that 15.5 million adults were living with DM and, of these, $7.0 \%$ originate from South Africa [2]. Diabetes mellitus is defined as a chronic metabolic disease characterized by prolonged hyperglycemia [3].

The prolonged hyperglycemia experienced by diabetic patients can result in macro- and microvascular complications that increases the risk for heart disease, stroke, and damage to the nervous system, retina, kidneys and other organs $[4,5]$. Therefore, DM treatment aims to maintain a blood glucose level within the physiological range [5]. Therapies implemented include dietary and lifestyle modification and the administration of oral anti diabetic drugs.

The preferred first line treatment in most clinical guidelines for the management of type 2 diabetes mellitus (T2DM), accounting for $\sim 90.0 \%$ of all DM cases, is metformin $[6,7]$. However, $38.0 \%$ of T2DM patients respond poorly to metformin [8]. In addition to biguanides, several 
other classes of drugs are being prescribed to treat T2DM; these include: sulfonylureas, meglitinides, thiazolidinediones, $\alpha$-glucosidase inhibitors, dipeptidyl peptidase- 4 inhibitors, glucagon-like peptide-1 agonist, sodium glucose cotransporter-2 inhibitors, insulin and its analogues [9-11].

Type 2 diabetes mellitus has been linked to variability in candidate genes that interfere with the management of glycemic control [9]. These candidate genes are involved in drug absorption, transportation, distribution, metabolism and the signaling cascade of oral anti diabetic drugs [12]. Studies have shown that the T2DM patient's response to treatment is characterized by inter-individual variability $[13,14]$. This variability in response have been linked to genetic and environmental factors $[15,16]$.

As metformin is the most common drug prescribed for the treatment and management of T2DM, numerous studies have been conducted to determine the therapeutic effects of metformin in the presence of genetic variants. Amongst the variants investigated, the $S L C$ variants feature quite often. Tzvetkov et al. [17] observed a variation in the renal clearance of metformin in Caucasian males with genetic polymorphisms in SLC22A1, SLC22A2 and SLC22A3. The renal transport of metformin was associated with a glucose lowering effect in combination with $S L C 47 A 1$ and $S L C 22 A 1$ genetic variants in a Dutch cohort [18]. Chen et al. [19] observed a very rare $S L C 22 A 1$ (R206C) variant in Asian patients diagnosed with T2DM. Patients with this rare variant demonstrated an altered response to metformin treatment. These studies and others like it, demonstrate the impact that $S L C$ variants and other genetic variants, have on the efficacy and toxicity of prescribed drugs.

Pharmacogenomic and pharmacokinetic studies have been conducted on the treatment response to T2DM in various populations across the world [20-23]. However, even though numerous studies have been conducted, limited data is available for sub-Saharan African populations and other African populations, regardless of the human genomic diversity found on this continent. Genetic diversity presented by indigenous populations across the world, in this instance South Africa, should be explored for improved diagnostic techniques and treatment plans for conditions such as diabetes, cardiovascular disease and cancer. The indigenous Nguni population of South Africa was selected for investigation in this study. The Nguni population is comprised of the Xhosa, Zulu, Ndebele and Swati clades [24-26].

Loci identified in previously studied populations observed anti diabetic drug efficacy may or may not affect efficacy in South African populations because of ethnic genetic differences. Seventeen single nucleotide polymorphism (SNP) biomarkers selected for investigation in this study, have previously been associated with T2DM in vari- ous populations across the world [17-23,27-28]. The aim of this study was to investigate the genetic association of these 17 SNP biomarkers and the response to anti diabetic treatment to determine their suitability for individualized metformin therapy in patients diagnosed with T2DM in the Nguni indigenous population of South Africa.

\section{MATERIALS AND METHODS}

Patients and Study Design. All participants were briefed about the project and a consent form was completed and submitted by each participant before the experiment was conducted. Ethics clearance for this study was obtained from the Senate Research Committee of the University of the Western Cape [Ethics clearance number BM/16/5/19].

Study Participants. A total of 140 T2DM outpatients belonging to the indigenous Nguni population of South Africa [Swati $(n=10)$, Xhosa $(n=81)$ and Zulu $(n=49)$ ] were recruited from the Cecilia Makhiwane Hospital (East London, Eastern Cape) and Piet Retief Hospital (Mkhondo, Mpumalanga). Type 2 diabetes mellitus, according to the WHO criteria of 1999: plasma glucose level between 7-13 $\mathrm{mmol} / \mathrm{L}$ with glycated hemoglobin $(\mathrm{Hb})$ level between 7.0 and $11.0 \%$. As some patients had other comorbidities (i.e. hypertension and dyslipidaemia) in this study, T2DM was diagnosed as a plasma glucose level between 6.0-27.0 $\mathrm{mmol} / \mathrm{L}$. Each patient participating in the study had $\mathrm{Hb} \mathrm{A}_{1 \mathrm{c}}$ levels measured within 6 month (baseline) and 12 month (follow-up) periods. Based on $\mathrm{Hb} \mathrm{A}_{1 \mathrm{c}}$ levels, patients were prescribed an average metformin dose of $1.95 \mathrm{mg}$ per day (with a maximum of $2.55 \mathrm{mg}$ ). Patients were categorized as controlled if they demonstrated a decreased $\mathrm{Hb} \mathrm{A}_{1 \mathrm{c}}$ value less than $8.0 \%$ at 12 months in comparison to the baseline prior to the study. Uncontrolled patients demonstrated an increased $\mathrm{Hb} \mathrm{\textrm {A } _ { 1 \mathrm { c } }}$ value more than $8.0 \%$ at 12 months in comparison to the baseline prior to the study. The classification used herein for controlled and uncontrolled T2DM has been described previously [29,30].

In this pool of study subjects, 53 patients demonstrated a controlled T2DM (responders to metformin therapy), with the remaining 87 patients demonstrated an uncontrolled T2DM (non-responders to metformin therapy). Patients were included in the study if they were 18 years or older and had been on treatment for at least 1 year prior to the study. All patients were on metformin monotherapy. Patients with other diseases such as type 1 diabetes mellitus (T1DM), malignancies, hyperlipidemia, chronic kidney and liver diseases, as well as pregnant patients, were excluded from the study. Information about age, family history, medical history, demographic parameters and medication used was obtained via medical reports and 
interviews. In addition to this, some patients were also on antihypertensive drugs, however, while the present study does not exclude drug-drug interactions, studies have not shown that other drugs co-administered with metformin have any influence on the outcome of a genetic association with metformin response.

Data Collection and Laboratory Measurements. A trained research nurse took clinical measurements of: weight, height and blood pressure (BP). Measurements were taken with all participants wearing minimal clothing and no shoes. Body mass index (BMI) for each patient was calculated as weight $(\mathrm{kg})$ divided by height $\left(\mathrm{m}^{2}\right)$ (Table 1$)$.

Random venous blood was collected to measure serum glycosylated $\mathrm{Hb}\left(\mathrm{Hb} \mathrm{A_{1c }}\right)$ levels. Furthermore, lipid profile [which includes: total cholesterol (TC), triglycerides (TG), low-density lipoprotein (LDL) and highdensity lipoprotein (HDL)] was obtained (Table 1). All blood samples were sent to relevant clinical laboratory centers for analysis.

Single Nucleotide Polymorphism(s) Selection and Genotyping. The 17 relevant pharmacogenomic variants selected for this study were chosen based upon previous publications, where association was made between SNPs and response to treatment with metformin. In addition to this, variants were also cross-referenced and selected based upon an evidence level ranging between 2B-4 dictated by the pharmacogenomics knowledge base, accessed on February 5 February 2019; PharmGKB (http://www.pharm gkb.org) [31].
Genomic DNA was isolated from buccal swabs using a standard salt lysis method [32]. Samples were stored at $-20{ }^{\circ} \mathrm{C}$. DNA was quantified using a NanoDrop ${ }^{\mathrm{TM}} 2000 /$ 2000c UV/VIS Spectrophotometer (Thermo Scientific, Waltham, MA, USA). The SNPs were genotyped using the MassARRAY ${ }^{\circledR}$ System IPLEX extension reaction (Agena Bioscience, San Diego, CA, USA). Genotypes of the selected SNP variants were determined for all the study participants (Table 2).

Statistical Analyses. Statistical analysis was performed using the Statistical Package for Social Sciences (SPSS) version 25 software (www.ibm.com/spss.statistics). Clinical laboratory data and anthropometric measurements were expressed as mean \pm SD. Hardy-Weinberg equilibrium (HWE) $p$ values were calculated for all SNPs using MedCalc version 2.2.0.0. (MedCalc Software, Ostend, Belgium), where $p$ value(s) of $<0.05$ were considered to be significant and implied that the population was not in HWE. Association between variant(s) and response to diabetic treatment was measured using odds ratios (ORs), $95 \%$ confidence interval $(95 \% \mathrm{CI})$ and $p$ value(s) derived from logistic regression. The threshold for significance in association studies was set at $p=0.05$.

\section{RESULTS}

Table 1 displays the clinical and biochemical demographics of the study population. All SNPs are within HWE with two SNPs (rs1801282 and rs6265) being mono-

Table 1. Clinical and biochemical demographics of the study population.

\begin{tabular}{|l|c|c|c|}
\hline Parameters & Controlled $(\boldsymbol{n}=\mathbf{5 3})$ & Uncontrolled $(\boldsymbol{n}=\mathbf{8 7})$ & $\boldsymbol{p}$ Value \\
\hline Sex $(\mathrm{F} ; \mathrm{M})$ & F: $36 ; \mathrm{M}: 14$ & F: $57 ;$ M: 30 & - \\
\hline Age $($ years $)$ & $60.7 \pm 11.0$ & $58.3 \pm 11.4$ & 0.470 \\
\hline Weight $(\mathrm{kg})$ & $85.8 \pm 19.5$ & $85.4 \pm 19.1$ & 0.833 \\
\hline Height $(\mathrm{cm})$ & $162.1 \pm 7.8$ & $163.1 \pm 7.7$ & 0.487 \\
\hline Body mass index & $31.9 \pm 8.4$ & $30.4 \pm 10.7$ & 0.304 \\
\hline Hb A $(\%)$ : & $7.6 \pm 2.0$ & $11.0 \pm 2.9$ & $<\mathbf{0 . 0 0 1}$ \\
baseline & $6.7 \pm 1.2$ & $11.5 \pm 2.9$ & $<\mathbf{0 . 0 0 1}$ \\
\hline Random blood sugar $(\%)$ & $9.4 \pm 3.9$ & $14.5 \pm 6.6$ & $<\mathbf{0 . 0 0 1}$ \\
\hline Systolic blood pressure $(\mathrm{mmHg})$ & $147.0 \pm 24.0$ & $90.3 \pm 13.7$ & 0.130 \\
\hline Diastolic blood pressure $(\mathrm{mmHg})$ & $83.9 \pm 15.5$ & $5.0 \pm 1.1$ & $\mathbf{0 . 0 1 8}$ \\
\hline Total cholesterol $(\mathrm{mmol} / \mathrm{L})$ & $4.4 \pm 1.1$ & $1.2 \pm 0.4$ & $\mathbf{0 . 0 0 1}$ \\
\hline High-density lipoprotein $(\mathrm{mmol} / \mathrm{L})$ & $1.2 \pm 0.4$ & $2.8 \pm 0.9$ & 0.672 \\
\hline Low-density lipoprotein $(\mathrm{mmol} / \mathrm{L})$ & $2.3 \pm 0.9$ & $2.3 \pm 1.1$ & $\mathbf{0 . 0 0 7}$ \\
\hline Triglycerides $(\mathrm{mmol} / \mathrm{L})$ & $2.0 \pm 1.2$ & $79.3 \pm 29.5$ & 0.215 \\
\hline Creatinine $(\mathrm{g} / \mathrm{mol})$ & $157.7 \pm 383.1$ & $52.6 \pm 25.0$ & 0.317 \\
\hline Glomerular filtration rate $\left(\mathrm{mL} / \mathrm{min} / 1.73 \mathrm{~m}^{2}\right.$ & $41.7 \pm 21.5$ & & 0.313 \\
\hline
\end{tabular}

Values are presented as mean \pm SD. Significant $p$ values $(<0.05)$ are bold. 
Table 2. Single nucleotide polymorphism information and Hardy-Weinberg p values in the study population.

\begin{tabular}{|c|c|c|c|c|c|c|}
\hline SNP & $\begin{array}{c}\text { Gene/ } \\
\text { Closest Gene }\end{array}$ & $\begin{array}{c}\text { Chromosomal } \\
\text { Position }\end{array}$ & Location & $\begin{array}{c}\text { Allele } \\
\text { Change }\end{array}$ & $\begin{array}{c}\text { Amino Acid } \\
\text { Change }\end{array}$ & $\begin{array}{c}\text { HWE } \\
p \text { Value }\end{array}$ \\
\hline rs10783050 & EEF1A1P11-RPL7P9 & $1: 96571527$ & intergenic & $\mathrm{T}>\mathrm{C}$ & - & 0.932 \\
\hline rs1143623 & $I L 1 B$ & $2: 112838252$ & intergenic & $\mathrm{C}>\mathrm{G}$ & - & 0.309 \\
\hline rs13266634 & SLC30A8 & $8: 117172544$ & missense & $\mathrm{C}>\mathrm{A} / \mathrm{T}$ & Arg325Trp & 0.532 \\
\hline rs13376631 & FMO1 & $1: 171266603$ & intron & $\mathrm{A}>\mathrm{G}$ & - & 0.903 \\
\hline rs1801282 & PPARG & $3: 12351626$ & missense & $\mathrm{C}>\mathrm{G}$ & Pro12Ala & monomorphic \\
\hline rs249429 & PRKAA1 & 5:40782137 & intron & $\mathrm{C}>\mathrm{T}$ & - & 0.299 \\
\hline rs 2815752 & NEGRI & $1: 72346757$ & intergenic & $G>A$ & - & 0.636 \\
\hline rs316009 & SLC22A2 & $6: 160254732$ & intron & $\mathrm{C}>\mathrm{T} / \mathrm{G}$ & - & 0.595 \\
\hline rs316019 & SLC22A2 & $6: 160249250$ & missense & $\mathrm{C}>\mathrm{A}$ & Ala270Ser & 0.808 \\
\hline rs391300 & SRR & $17: 2312964$ & intron & $\mathrm{C}>\mathrm{T}$ & - & 0.739 \\
\hline rs461473 & SLC22A1 & $6: 160122530$ & intron & $\mathrm{G}>\mathrm{A}$ & - & 0.898 \\
\hline rs4810083 & PCK1 & 20:57545215 & intergenic & $\mathrm{C}>\mathrm{T}$ & - & 0.145 \\
\hline rs578427 & - & $6: 91702432$ & intergenic & $\mathrm{T}>\mathrm{C}$ & - & 0.909 \\
\hline rs622342 & SLC22A1 & $6: 160151834$ & intron & $A>C$ & - & 0.218 \\
\hline rs6265 & $B D N F ; B D N F-A S$ & $11: 27658369$ & missense & $\mathrm{C}>\mathrm{T}$ & Val66Met & monomorphic \\
\hline rs819 2675 & SLC2A2 & $3: 171007094$ & intron & $\mathrm{C}>\mathrm{T}$ & - & 0.674 \\
\hline
\end{tabular}

Table 3. Genotype and allele frequencies of 13 single nucleotide polymorphism(s) demonstrating no significant association to type 2 diabetes mellitus treatment response.

\begin{tabular}{|c|c|c|c|c|c|}
\hline SNP & $\begin{array}{l}\text { Genotype/ } \\
\text { Allele }\end{array}$ & $\begin{array}{c}\text { Control } \\
n(\%)\end{array}$ & $\begin{array}{c}\text { Uncontrolled } \\
n(\%)\end{array}$ & OR $(95 \%$ CI $)$ & $p$ Values \\
\hline rs 10783050 & $\begin{array}{l}\text { TT } \\
\text { TC } \\
\text { T } \\
\text { C }\end{array}$ & $\begin{aligned} & 52(98.1) \\
& 1(1.9) \\
& 105(99.1) \\
& 1(0.9)\end{aligned}$ & $\begin{array}{c}86(98.9) \\
0(0.0) \\
172(98.9) \\
0(0.0)\end{array}$ & $\begin{array}{l}\text { reference } \\
0.20(0.01-5.06) \\
\text { reference } \\
0.20(0.01-5.05)\end{array}$ & $\begin{array}{l}0.331 \\
0.332\end{array}$ \\
\hline rs1143623 & $\begin{array}{l}\mathrm{CC} \\
\mathrm{CG} \\
\mathrm{C} \\
\mathrm{G}\end{array}$ & $\begin{aligned} & 48(90.6) \\
& 5(9.4) \\
& 96(90.6) \\
& 10(9.4)\end{aligned}$ & $\begin{array}{r}74(85.1) \\
11(12.6) \\
159(91.4) \\
11(6.3)\end{array}$ & $\begin{array}{l}\text { reference } \\
1.43(0.47-4.36) \\
\text { reference } \\
0.66(0.27-1.62)\end{array}$ & $\begin{array}{l}0.533 \\
0.369\end{array}$ \\
\hline rs13266634 & $\begin{array}{l}\mathrm{CC} \\
\mathrm{CT} \\
\mathrm{C} \\
\mathrm{T}\end{array}$ & $\begin{array}{c}50(94.3) \\
3(5.7) \\
100(94.3) \\
6(5.7)\end{array}$ & $\begin{array}{c}78(89.7) \\
8(9.2) \\
164(94.3) \\
8(4.6)\end{array}$ & \begin{tabular}{|l|} 
reference \\
$1.71(0.43-6.75)$ \\
reference \\
$0.81(0.27-2.41)$
\end{tabular} & $\begin{array}{l}0.444 \\
0.709\end{array}$ \\
\hline rs13376631 & $\begin{array}{l}\text { AA } \\
\text { GG } \\
\text { AG } \\
\text { A } \\
\text { G } \\
\end{array}$ & $\begin{array}{l}16(30.2) \\
11(20.8) \\
25(13.3) \\
57(53.8) \\
47(44.3)\end{array}$ & $\begin{array}{l}22(25.3) \\
17(19.5) \\
37(42.5) \\
81(46.6) \\
71(40.8)\end{array}$ & $\begin{array}{l}\text { reference } \\
1.12(0.42-3.04) \\
1.08(0.47-2.44) \\
\text { reference } \\
1.06(0.64-1.75)\end{array}$ & $\begin{array}{l}0.818 \\
0.860 \\
0.811\end{array}$ \\
\hline rs1801282 & $\begin{array}{l}\mathrm{CC} \\
\mathrm{C}\end{array}$ & $\begin{array}{r}53(100.0) \\
106(100.0)\end{array}$ & $\begin{array}{r}86(98.9) \\
172(98.9)\end{array}$ & $\begin{array}{l}\text { monomorphic } \\
-\end{array}$ & monomorphic \\
\hline rs249429 & $\begin{array}{l}\text { TT } \\
\text { CC } \\
\text { CT } \\
\text { T } \\
\text { C }\end{array}$ & $\begin{array}{c}28(52.8) \\
3(5.7) \\
22(41.5) \\
78(73.6) \\
28(26.4)\end{array}$ & $\begin{array}{c}40(46.0) \\
6(6.9) \\
40(46.0) \\
120(69.0) \\
52(29.9)\end{array}$ & $\begin{array}{l}\text { reference } \\
1.40(0.32-6.07) \\
1.27(0.6258-2.5885) \\
\text { reference } \\
1.21(0.70-2.07)\end{array}$ & $\begin{array}{l}0.650 \\
0.510 \\
0.500\end{array}$ \\
\hline rs2815752 & $\begin{array}{l}\text { GG } \\
\text { AA } \\
\text { GA } \\
\text { G } \\
\text { A }\end{array}$ & $\begin{array}{l}18(34.0) \\
13(24.5) \\
22(41.5) \\
58(54.7) \\
48(45.2)\end{array}$ & $\begin{array}{l}22(25.3) \\
20(23.0) \\
43(49.4) \\
87(50.0) \\
83(47.7)\end{array}$ & $\begin{array}{l}\text { reference } \\
1.26(0.49-3.21) \\
1.60(0.71-3.59) \\
\text { reference } \\
1.15(0.71-3.59)\end{array}$ & $\begin{array}{l}0.630 \\
0.254 \\
0.567\end{array}$ \\
\hline
\end{tabular}


BALKAN JOURNAL OF MEDICAL GENETICS

Abrahams-October Z, Xhakaza L, Pearce B, Mandisa Masilela C, Benjeddou M, Vincent Adeniyi O, Johnson R, Jebio Ongole J

Continuation of the previous page

\begin{tabular}{|c|c|c|c|c|c|}
\hline SNP & $\begin{array}{l}\text { Genotype/ } \\
\text { Allele }\end{array}$ & $\begin{array}{c}\text { Controlled } \\
n(\%)\end{array}$ & $\begin{array}{c}\text { Uncontrolled } \\
n(\%)\end{array}$ & OR $(95 \%$ CI $)$ & $p$ Values \\
\hline rs34834489 & $\begin{array}{l}\text { GG } \\
\text { GA } \\
\text { G } \\
\text { A }\end{array}$ & $\begin{array}{c}46(86.7) \\
6(11.3) \\
98(92.5) \\
6(5.7)\end{array}$ & $\begin{array}{c}80(92.0) \\
6(6.9) \\
166(95.4) \\
6(3.4)\end{array}$ & $\begin{array}{l}\text { reference } \\
0.58(0.18-1.89) \\
\text { reference } \\
0.59(0.19-1.88)\end{array}$ & $\begin{array}{l}0.361 \\
0.373\end{array}$ \\
\hline rs 391300 & $\begin{array}{l}\mathrm{CC} \\
\mathrm{TT} \\
\mathrm{CT} \\
\mathrm{C} \\
\mathrm{T}\end{array}$ & $\begin{array}{r}9(17.0) \\
18(34.0) \\
26(49.1) \\
44(41.5) \\
62(58.5)\end{array}$ & $\begin{array}{l}17(19.5) \\
24(27.6) \\
44(50.6) \\
78(44.8) \\
92(52.9)\end{array}$ & $\begin{array}{l}\text { reference } \\
0.71(0.26-1.94) \\
0.90(0.35-02.30) \\
\text { reference } \\
0.84(0.51-1.37)\end{array}$ & $\begin{array}{l}0.500 \\
0.819 \\
0.477\end{array}$ \\
\hline rs461473 & $\begin{array}{l}\text { GG } \\
\text { GA } \\
\text { G } \\
\text { A }\end{array}$ & $\begin{array}{c}52(98.1) \\
1(1.9) \\
105(99.1) \\
1(0.9)\end{array}$ & $\begin{array}{c}84(96.6) \\
2(2.3) \\
170(97.7) \\
2(1.1)\end{array}$ & $\begin{array}{l}\text { reference } \\
1.24(0.11-14.00) \\
\text { reference } \\
1.24(0.11-13.99)\end{array}$ & $\begin{array}{l}0.863 \\
0.864\end{array}$ \\
\hline rs622342 & $\begin{array}{l}\mathrm{AA} \\
\mathrm{CC} \\
\mathrm{CA} \\
\mathrm{A} \\
\mathrm{C}\end{array}$ & $\begin{array}{c}33(62.3) \\
3(5.7) \\
18(34.0) \\
84(79.2) \\
22(20.8)\end{array}$ & $\begin{array}{r}48(55.2) \\
12(13.8) \\
26(29.9) \\
122(70.1) \\
50(28.7)\end{array}$ & $\begin{array}{l}\text { reference } \\
4.13(0.87-19.65) \\
0.99(0.472 .10) \\
\text { reference } \\
1.56(0.88-2.78)\end{array}$ & $\begin{array}{l}0.075 \\
0.985 \\
0.113\end{array}$ \\
\hline rs6265 & $\begin{array}{l}\mathrm{CC} \\
\mathrm{C} \\
\end{array}$ & $\begin{array}{r}53(100.0) \\
106(100.0) \\
\end{array}$ & $\begin{array}{r}86(98.9) \\
172(98.9) \\
\end{array}$ & $\begin{array}{l}\text { monomorphic } \\
-\end{array}$ & $\begin{array}{l}\text { monomorphic } \\
-\end{array}$ \\
\hline rs8192675 & $\begin{array}{l}\text { CC } \\
\text { TT } \\
\text { CT } \\
\text { C } \\
\text { T }\end{array}$ & $\begin{array}{c}42(79.2) \\
1(1.9) \\
9(17.0) \\
93(87.7) \\
11(10.4)\end{array}$ & $\begin{array}{c}58(66.7) \\
3(3.4) \\
25(28.7) \\
141(81.0) \\
31(17.8\end{array}$ & $\begin{array}{l}\text { reference } \\
2.17(0.22-21.62) \\
2.01(0.85-4.75) \\
\text { reference } \\
1.86(0.89-3.88)\end{array}$ & $\begin{array}{l}0.508 \\
0.111 \\
0.099\end{array}$ \\
\hline
\end{tabular}

OR: odds ratio; 95\% CI: 95\% confidence interval.

Percent does not account for missing allele(s) at specific loci.

Table 4. Genotype and allele frequencies of four single nucleotide polymorphism(s) demonstrating no significant association to type 2 diabetes mellitus treatment response.

\begin{tabular}{|c|c|c|c|c|c|c|c|c|}
\hline & & & & Unadjust & & Adjustec & & \\
\hline SNP & $\begin{array}{l}\text { Genotype/ } \\
\text { Allele }\end{array}$ & $\begin{array}{c}\text { Controlled } \\
n(\%)\end{array}$ & $\begin{array}{c}\text { Uncontrolled } \\
n(\%)\end{array}$ & OR (95\% CI) & $p$ Values & OR (95\% CI) & $p$ Values & $\begin{array}{l}\text { Bonferonni } \\
\text { Corrected } p \text { Values }\end{array}$ \\
\hline rs316009 & $\begin{array}{l}\text { CC } \\
\text { CT } \\
\text { C } \\
\mathrm{T}\end{array}$ & $\begin{array}{r}45(84.9) \\
8(15.1) \\
98(92.5) \\
8(0.08)\end{array}$ & $\begin{array}{r}83(95.4) \\
3(0.03) \\
169(97.1) \\
3(0.02)\end{array}$ & $\begin{array}{l}\text { reference } \\
0.20(0.05-0.81) \\
\text { reference } \\
0.22(0.06-0.84)\end{array}$ & $\begin{array}{l}0.023 \\
0.027\end{array}$ & \begin{tabular}{l|} 
reference \\
$0.31(0.05-0.190)$ \\
reference \\
$0.85(0.01-0.93)$
\end{tabular} & $\begin{array}{l}0.204 \\
\mathbf{0 . 0 4 4}\end{array}$ & $\begin{array}{l}- \\
0.088\end{array}$ \\
\hline rs316019 & $\begin{array}{l}\text { CC } \\
\text { AA } \\
\text { CA } \\
\text { C } \\
\text { A }\end{array}$ & $\begin{array}{r}41(77.4) \\
1(0.02) \\
11(20.8) \\
93(87.7) \\
13(12.3)\end{array}$ & $\begin{array}{c}77(88.5) \\
0(0.0) \\
8(0.09) \\
162(93.1) \\
8(0.05)\end{array}$ & $\begin{array}{l}\text { reference } \\
0.18(0.01-4.48) \\
0.39(0.14-1.04) \\
\text { reference } \\
0.35(0.14-0.8)\end{array}$ & $\begin{array}{l}0.295 \\
0.059 \\
\\
\mathbf{0 . 0 2 6}\end{array}$ & $\begin{array}{l}\text { reference } \\
- \\
- \\
\text { reference } \\
0.72(0.21-2.44)\end{array}$ & 0.593 & $\begin{array}{l}- \\
- \\
-\end{array}$ \\
\hline rs4810083 & $\begin{array}{l}\text { CC } \\
\text { TT } \\
\text { CT } \\
\text { C } \\
\text { T } \\
\end{array}$ & $\begin{array}{l}16(30.2) \\
10(18.9) \\
27(35.7) \\
59(55.7) \\
47(44.3)\end{array}$ & $\begin{array}{l}33(37.9) \\
32(36.9) \\
21(43.1) \\
75(43.1) \\
97(55.7)\end{array}$ & $\begin{array}{l}\text { reference } \\
1.55(0.61-3.92) \\
0.38(0.17-0.86) \\
\text { reference } \\
1.62(1.00-2.64)\end{array}$ & $\begin{array}{r}0.353 \\
\mathbf{0 . 0 2 1} \\
\\
0.051 \\
\end{array}$ & $\begin{array}{l}\text { reference } \\
1.24(0.42-3.60) \\
2.80(1.01-7.79) \\
\text { reference } \\
0.81(0.48-1.36)\end{array}$ & $\begin{array}{r}0.698 \\
\mathbf{0 . 0 4 9} \\
\\
0.423 \\
\end{array}$ & $\begin{array}{l}- \\
0.098 \\
- \\
\end{array}$ \\
\hline rs578427 & $\begin{array}{l}\text { TT } \\
\text { CC } \\
\text { CT } \\
\text { T } \\
\text { C }\end{array}$ & $\begin{array}{c}10(18.9) \\
4(7.5) \\
39(73.6) \\
47(44.3) \\
59(55.7)\end{array}$ & $\begin{array}{l}15(17.2) \\
28(32.2) \\
43(49.4) \\
73(42.0) \\
99(56.9)\end{array}$ & $\begin{array}{l}\text { reference } \\
4.67(1.25-17.44) \\
0.74(0.30-1.83) \\
\text { reference } \\
1.08(0.66-1.76)\end{array}$ & $\begin{array}{l}\mathbf{0 . 0 2 2} \\
0.507 \\
0.765\end{array}$ & $\begin{array}{l}\text { reference } \\
1.80(0.58-5.64) \\
1.70(0.45-2.55) \\
\text { reference } \\
1.31(0.78-2.22)\end{array}$ & $\begin{array}{l}0.312 \\
0.884 \\
0.308\end{array}$ & $\begin{array}{l}- \\
- \\
-\end{array}$ \\
\hline
\end{tabular}

OR: odds ratio; $95 \% \mathrm{CI}$ : 95\% confidence interval.

Percent does not account for missing allele(s) at specific loci. Significant $p$ values $(<0.05)$ are bold. 
morphic (Table 2). Hardy-Weinberg equilibrium $p$ values ranged between 0.145-0.932 for all of the studied SNPs in the population. Genotype and allele distribution of the 17 SNPS were determined in all the study participants (Table 3). Among the SNPs selected for this study, four displayed significant association between T2DM and/or genotype or allele frequencies prior to adjustment (Table 4). The four significantly associated SNPs identified are: rs316009, rs316019, rs4810083 and rs578427. Prior to adjustment, the heterozygous genotype, i.e. $\mathrm{CT}$, and the minor allele $\mathrm{T}$ of rs316009 demonstrated significant association between T2DM and treatment response $[p=0.023$ (OR: $0.20 ; 95 \% \mathrm{Cl}: 0.05-0.81$ ) and $p=0.027$ (OR: $0.22 ; 95 \%$ Cl: 0.06-0.84) respectively] (Table 4). The minor A allele of rs316019 with a $p$ value of 0.026 (OR: $0.35 ; 95 \% \mathrm{Cl}$ : 0.14-0.88) also showed a significant association (Table 4). The heterozygous genotype, i.e. $\mathrm{CT}$ of rs 4810083 with a $p$ value of 0.021 (OR: 0.38 ; $95 \% \mathrm{Cl}: 0.17-0.86$ ) and the homozygous minor genotype CC of rs578427 with a $p$ value of 0.022 (OR: $4.67 ; 95 \% \mathrm{Cl}: 1.25-1.83$ ) also demonstrated a significant association between T2DM and response (Table 4). However, after adjustment, only the T allele of rs316009 with a $p=0.044$ (OR: $0.85 ; 95 \% \mathrm{Cl}$ : 0.01-0.93) and the CT genotype of rs4810083 with a $p=0.049$ (OR: $2.80 ; 95 \% \mathrm{Cl}: 1.01-7.79)$ could still be associated with a response to treatment. Lastly, after Bonferroni correction, both rs316009 with a $p=0.088$ and rs4810083 with a $p=$ 0.098, showed a lack of association.

\section{DISCUSSION}

In this study the genetic association of 17 pharmacogenomic biomarkers and response to metformin treatment in the indigenous Nguni population of South Africa was determined. Previously, the MATE2K variant, rs 12943590 and the variant rs 12752688 , had been suggested for inclusion in pharmacogenomic profiling of the Nguni population [24]. This study will provide additional pharmacogenomic biomarker information about possible associations between genetic variants and response to metformin therapy in the Nguni population.

All SNPs, besides rs1801282 and rs6265 (which were shown to be monomorphic), were within HWE and showed $p$ values ranged between $0.145-0.932$ in the study population (Table 2). The two monomorphic SNPs (i.e. rs 1801282 and rs6265) are rare variants, however, they were included in the study because of the important roles they play in the development and progression of the diabetes disease.

The PPARG variant, rs1801282, is important in the development of obesity as well as adipose and muscle tissue metabolism [33]. This variant has recently been investigated in the development of early visual impairment in T2DM Chinese Han population [33] and been associated with obstructive sleep apnea in Chinese Han and Indian subjects diagnosed with T2DM [34,35]. Obesity is a known comorbid disease of diabetes and sleep apnea has also been associated with diabetes, therefore, this variant was included for investigation.

The $B D N F$ gene theoretically plays a significant role on the well-being and health of individuals, as it has diverse roles throughout the body and brain [36]. The BDNF variant, rs6265, has been linked to obesity and T2DM in Chinese populations [36,37] and BMI in Korean [38] and British populations [39]. Because this variant could affect T2DM, comorbid diseases related to diabetes and other physical indicators of the progression of diabetes, it was selected for the study, regardless of its rarity in African populations.

Genotype and allele distribution of the 17 SNPs were determined in all the study participants (Tables 3 and 4). Among the SNPs analyzed, 13 of the SNPs selected for this study showed no statistically significant association between treatment response and the SNP variant (Table 3). The remaining four variants however, i.e. $\mathrm{rs} 316009$ (genotype $p$ value 0.023 ; allele $p$ value 0.027 ), rs316019 (genotype $p$ value 0.026 ), rs4810083 (genotype $p$ value 0.021 ) and rs578427 (genotype $p$ value 0.022 ), showed a significant association between variant and treatment response prior to adjustment (Table 4). This study showed an increased treatment response to metformin for T2DM patients with SNP variants rs316009, rs316019 and rs4810083. In contrast, rs578427 demonstrated a decrease in response to treatment. However, post adjustment, only the T allele of rs316009 ( $p$ value 0.044 ) and the CT genotype of rs4810083 ( $p$ value 0.049) were associated with treatment response. Post Bonferroni correction rs316009 ( $p$ value 0.088 ) and rs4810083 ( $p$ value 0.098 ), demonstrated a lack of association. However, this can be attributed to the small sample size of the study cohort.

The rs 316009 variant is located in a transcription factor binding motif and is in linkage disequilibrium with the non synonymous variant rs316019 [21,40-47]. In previous studies, the TT genotype of rs316009 showed an increase response to metformin in comparison to the $\mathrm{CC}$ and $\mathrm{CT}$ genotypes [41]. Unfortunately, the homozygous TT genotype was not observed in this study population. From the data available, the CT genotype demonstrated a better response to treatment in comparison to the CC genotype (Table 4). The rs316019 is the most common variant of SLC22A2 in many populations and has displayed contradictory results, linked to both decreased and increased renal clearance of metformin in healthy subjects [5,40,42-45].

The interaction of metformin and other drugs in the presence of rs316019 was determined in silico by Sajib et al. [43]. Based upon the in silico data generated by Sajib et al. 
[43], all substrates bind to the same pocket of SLC22A2 and substrates fit better to the binding site of the C allele [43]. The rs316019 results in a protein change that clears metformin from circulation much more slowly than the wild-type [43]. The AA genotype, especially in females, has been linked to hyperlactacidemia within clinical doses of metformin [43]. Thus, dose adjustments based on the rs316019 variant may be beneficial to maximize treatment response.

Prior to correction, the A allele was significantly associated with an improved response to treatment. This is in contradiction to studies conducted by Song et al. [44] and Wang et al. [42], as well as the in silico data generated by Sajib et al. [43]. This data is however in agreement with studies conducted by Chen et al. [40]. Other studies also indicated no association between this variant and response to metformin treatment [17,21].

The SNP variant rs4810083 T allele is not associated with a response to metformin treatment in T2DM patients [46]. The results obtained in this study, however, may suggest that the $\mathrm{T}$ allele is most likely to be associated with a decrease in response to diabetic treatment as more patients in the uncontrolled category carry the T allele in comparison to the controlled category. This study group also shows the CT genotype to be associated with an improved response to treatment (Table 4). To enable further clarity with regard to the significance of this SNP variant, more data is required from other population groups as well as a bigger sample cohort for the current study group.

In the case of the SNP rs578427, the TT genotype has been associated with an increased renal clearance and secretion clearance of metformin in comparison to the CC genotype in a healthy population [47]. As the accumulation of metformin in the body can result in the development of lactic acidosis, the TT genotype can thus be associated with an improved response to treatment. These results are in concordance with the data generated for this study population as the CC genotype was shown to be significantly associated with a decreased response to treatment (Table 4).

Contradictory, as well as inconclusive, results may have arisen for a number of reasons. The most relevant being sample size as well as SNP selection and the approach used to analyze individual SNPs. Because SNPs do not occur in isolation of each other, but rather as combinations forming defined haplotypes, the phenotypic effect of individual SNPs is not always consistent with functional effects. Thus, genotyping single or a few individual SNPs may fail to reflect the true functionality of genetic variants [48]. Therefore, it should be recommended that future studies evaluate haplotypes to establish the functional effects that a collection of SNPs may have on response to treatment.

Conclusions. In this study, two SNP variants (rs316009 and rs4810083) were significantly associated with improved response to diabetic treatment prior to Bonferroni correction. The greatest limitation of this study was the sample size and this has inadvertently affected the relevance of significantly associated SNP variants. Regardless of this, this study provides additional important data regarding possible associations between genetic variants and metformin therapy outcomes. In, addition, this study is one of the first studies providing genetic data from the understudied indigenous sub-Saharan African populations.

Acknowledgments. The authors would like to thank the study participants, Cecilia Makiwane Hospital and the Department of Health Eastern Cape (Mdantsane, Eastern Cape, South Africa). The content hereof is the sole responsibility of the authors and do not necessarily represent the official views of the South African Medical Research Council or the funders.

Declaration of Interest. The authors report no conflicts of interest. The authors alone are responsible for the content and writing of this article.

Funding. The study reported herein was made possible through funding by the South African Medical Research Council through its Division of Research Capacity Development under funding received from the South African National Treasury (Cape Town, Western Cape, South Africa). In addition, partial funding from the National Research Foundation of South African and the University of the Western Cape was used for this study.

\section{REFERENCES}

1. Ogurtsova K, da Rocha Fernandes JD, Huang Y, Linnenkamp U, Guariguata L, Cho NH, et al. IDF Diabetes Atlas: Global estimates for the prevalence of diabetes for 2015 and 2040. Diabetes Res Clin Pract. 2017; 128: 40-50.

2. International Diabetes Federation. IDF Diabetes Atlas 8th ed., 2017. Brussels, Belgium. (Available at: https:// diabetesatlas.org [accessed: July 15 2018].

3. Hoogwerf BJ, Sferra J, Donley BG. Diabetes mellitus-overview. Foot Ankle Clin. 2006; 11(4): 703-715.

4. Chawla A, Chawla R, Jaggi S. Microvascular and macrovascular complications in diabetes mellitus: Distinct or continuum? Indian J Endocrinol Metab. 2016; 20(4): 546-551.

5. Islam T, Rahman MS, Paul N, Akhteruzzaman S, Sajib AA. Allele-specific detection of SLC22A2 rs316019 variants associated with metformin disposition through the kidney. Int J Diabetes and Metab. 2018; 24: 22-28. 
6. American Diabetes Association (ADA). Diagnosis and classification of diabetes mellitus. Diabetes Care. 2014; 37(Suppl 1): S81-S90.

7. World Health Organization. WHO model list of essential medicines (20th list) [accessed: October 5 2018].

8. Inzucchi SE, Lipska KJ, Mayo H, Bailey CJ, McGuire DK. Metformin in patients with type 2 diabetes and kidney disease: A systematic review. JAMA. 2014; 312(24):2668-2675.

9. Singh S, Usman K, Banerjee M. Pharmacogenetic studies update in type 2 diabetes mellitus. World J Diabetes. 2016; 7(15): 302-315.

10. Inzucchi SE, Bergenstal RM, Buse JB, Diamant M, Ferrannini E, Nauck M, et al. Management of hyperglycaemia in type 2 diabetes: A patient-centered approach. Position statement of the American Diabetes Association (ADA) and the European Association for the Study of Diabetes (EASD). Diabetologia. 2012; 55(6): 1577-1596.

11. Topić E. The role of pharmacogenetics in the treatment of diabetes mellitus. J Med Biochem. 2014; 33(1): 58-70.

12. Mannino GC, Sesti G. Individualized therapy for type 2 diabetes: Clinical implications of pharmacogenetic data. Mol Diagn Ther. 2012; 16(5): 285-302.

13. Hermann LS, Scherstén B, Bitzén PO, Kjellström T, Lindgärde, F, Melander A. Therapeutic comparison of metformin and sulfonylurea, alone and in various combinations: A double-blind controlled study. Diabetes Care. 1994; 17(10): 1100-1109.

14. Cook MN, Girman CJ, Stein PP, Alexander CM. Initial monotherapy with either metformin or sulphonylureas often fails to achieve or maintain current glycaemic goals in patients with type 2 diabetes in UK primary care. Diabet Med. 2007; 24(4): 350-358.

15. Chen L, Pei JH, Kuang J, Chen HM, Chen Z, Li ZW, et al. Effect of lifestyle intervention in patients with type 2 diabetes: A meta-analysis. Metabolism. 2015; 64(2): 338-347.

16. Zia A, Kiani AK, Bhatti A, John P. Genetic susceptibility to type 2 diabetes and implications for therapy. J Diabetes Metab. 2013; 4(3): 248.

17. Tzvetkov MV, Vormfelde SV, Balen D, Meineke I, Schmidt T, Sehrt D, et al. The effects of genetic polymorphisms in the organic cation transporters OCT1, OCT2, and OCT3 on the renal clearance of metformin. Clin Pharmacol Ther. 2009; 86(3): 299-306.

18. Becker ML, Visser LE, Van Schaik RHN, Hofman A, Uitterlinden AG, Stricker BHC. Genetic variation in the multidrug and toxin extrusion 1 transporter protein influences the glucose-lowering effect of metformin in patients with diabetes: A preliminary study. Diabetes. 2009; 58(3): 745-749.

19. Chen L, Takizawa M, Chen E, Schlessinger A, Segenthelar J, Choi JH, et al. Genetic polymorphisms in organic cation transporter 1 (OCT1) in Chinese and Japanese populations exhibit altered function. J Pharmacol Exp Ther. 2010; 335(1): 42-50.

20. Sanchez-Ibarra HE, Reyes-Cortes LM, Jiang XL, LunaAguirre CM, Aguirre-Trevino D, Morales-Alvarado IA, et al. Genotypic and phenotypic factors influencing drug response in Mexican patients with type 2 diabetes mellitus. Front Pharmacol. 2018; 9: 320.

21. Christensen MMH, Pedersen RS, Stage TB, BraschAndersen C, Nielsen F, Damkier P, et al. A gene-gene interaction between polymorphisms in the OCT2 and MATE1 genes influences the renal clearance of metformin. Pharmacogenet Genomics. 2013; 23(10): 526-534.

22. Shokri F, Ghaedi H, Fard SG, Movafagh A, Abediankenari S, Mahrooz A, et al. Impact of ATM and SLC22A1 polymorphisms on therapeutic response to met-formin in Iranian diabetic patients. Int J Mol Cell Med. 2016; 5(1): 1-7.

23. Iskakova AN, Romanova AA, Aitkulova AM, Sikhayeva NS, Zholdybayeva EV, Ramanculov EM. Poly-morphisms in genes involved in the absorption, distribution, metabolism, and excretion of drugs in the Kazakhs of Kazakhstan. BMC Genet. 2016; 17: 23.

24. Xhakaza L, Abrahams-October Z, Pearce B, Masilela CM, Adeniyi OV, Johnson R, et al. Evaluation of the suitability of 19 pharmacogenomics biomarkers for individualized metformin therapy for type 2 diabetes patients. Drug Metabol Pers Ther. 2020; 35(2): j/ dmdi. 2020.35.issue-2/dmpt-2020-0111/dmpt-20200111.xml. doi: 10.1515/dmpt-2020-0111.

25. Lucassen A, Ehlers K, Grobler P, Shezi AL. Allele frequency data of 14 autosomal STR loci in four major population groups of South Africa. Int J Legal Med. 2018; 128(2): 275-276.

26. Lane AB, Soodyall H, Arndt S, Ratshikhopha ME, Jonker E, Freeman C, et al. Genetic substructure in South African Bantu-speakers: Evidence from autosomal DNA and Y-chromosome studies. Am J Phys Anthropol. 2002; 119(2): 175-185.

27. Xiao D, Zhang S, Li X, Yin J, Gong W, Zheng Y, et al. IL-1B rs1143623 and EEF1A1P11-RPL7P9 rs 10783050 polymorphisms affect the glucose-lowing efficacy of metformin in Chinese overweight or obese 
type 2 diabetes mellitus patients. Pharmacogenomics. 2015; 16(14): 1621-1629.

28. Omori S, Tanaka Y, Takahashi A, Hirose H, Kashiwagi A, Kaku K, et al. Association of CDKAL1, IGF2BP2, CDKN2A/B, HHEX, SLC30A8, and KCNJ11 with susceptibility to type 2 diabetes in a Japanese population. Diabetes. 2008; 57(3): 791-795.

29. Kashki Z, Masoumi P, Mahrooz A, Hashemi-Soteh MB, Bahar A, Alizadeh A. The variant organic cation transporter 2 (OCT2)-T201M contribute to changes in insulin resistance in patients with type 2 diabetes treated with metformin. Diabetes Res Clin Pract. 2015; 108(1): 78-83.

30. Clinical guidelines (CDE). Available at: http://www. cdediabetes.co.za [accessed: October 30 2018].

31. Pharmacogenomic knowledge base (PharmGKB). Available at: http://www.pharmgkb.org [accessed: July 5 2018].

32. Leat N, Benjeddou M, Davison S. Nine-locus Y-chromosome STR profiling of Caucasian and Xhosa populations from Cape Town, South Africa. Forensic Sci Int. 2004; 144(1): 73-75.

33. Li T, Xu X, Xu Y, Jin P, Chen J, Shi Y, et al. PPARG Polymorphisms are associated with unexplained mild vision loss in patients with type 2 diabetes mellitus. J Opthalmol. 2019; 2019: 5284867. doi: 10.1155/2019/ 5284867. eCollection 2019.

34. Jiao X, Yang S, Yang Y, Li J, Sun H, Zhang M, et al. Targeted sequencing analysis of PPARG identifies a risk variant associated with obstructive sleep apnea in Chinese Han subjects. Sleep Breath. 2020; 24(1): 167-174.

35. Bhushan B, Guleriab R, Misrac A, Luthrad K, Kumare G. Association of PPAR $\gamma 2$ (Pro12Ala) and neuropeptide Y (Leu7Pro) gene polymorphisms with obstructive sleep apnea in obese Asian Indians. Dis Markers. 2011; 30(1): 31-38.

36. Zhou J-X, Li H-C, Bai X-J, Chang B-C, Li C-J, Sun $\mathrm{P}$, et al. Functional Val66Met polymorphism of brainderived neurotrophic factor in type 2 diabetes with depression in Han Chinese subjects. Behav Brain Funct. 2013; 9: 34.

37. Wu L, Xi B, Zhang M, Shen Y, Zhao X, Cheng H, et al. Associations of six single nucleotide polymorphisms in obesity related genes with BMI and risk of obesity in Chinese children. Diabetes. 2010; 59(12): 3085-3089.

38. Hong K, Lim J, Go M, Cho Y, Ahn Y, Han B, et al. Recapitulation of the association of Val66Met polymorphism of BDNF gene with BMI in Koreans. Obesity. 2012; 20(9): 1871-1875.
39. Shugart Y, Chen L, Day I, Lewis S, Timpson S, Yuan $\mathrm{W}$, et al. Two British women studies replicated the association between the Val66Met polymorphism in the brain-derived neurotrophic factor (BDNF) and BMI. Eur J Hum Genet. 2009; 17(8): 1050-1055.

40. Chen Y, Li S, Brown C, Cheatham S, Castro RA, Leabman MK, et al. Effect of genetic variation in the organic cation transporter 2 on the renal elimination of metformin. Pharmacogenet Genomics. 2009; 19(7): 497-504.

41. Goswami S, Yee SW, Xu F, Sridhar SB, Mosley JD, Takahashi A, et al. A longitudinal HbA1c model elucidates genes linked to disease progression on metformin. Clin Pharmacol Ther. 2016; 100(5): 537-547.

42. Wang ZJ, Yin OQP, Tomlinson B, Chow MSS. OCT2 Polymorphisms and in-vivo renal functional consequence: Studies with metformin and cimetidine. Pharmacogenet Genomics. 2008; 18(7): 637-645.

43. Sajib AA, Islam T, Paul N, Yeasmin S. Interaction of rs316019 variants of SLC22A2 with metformin and other drugs-an in silico analysis. J Genet Eng Biotechnol. 2018; 16(2): 769-775.

44. Song IS, Shin HJ, Shim EJ, Jung IS, Kim WY, Shon $\mathrm{JH}$, et al. Genetic variants of the organic cation transporter 2 influence the disposition of metformin. Clin Phar-macol Ther. 2008; 84(5):559-562.

45. Zolk O, Solbach TF, König J, Fromm MF. Functional characterization of the human organic cation transporter 2 variant p.270Ala $>$ Ser. Drug Metab Dispos. 2009; 37(6): 1312-1318.

46. Tkac I, Javorsky M, Klimcakova L, Zidzik J, Gala I, Babjakova $\mathrm{E}$, et al. A pharmacogenetic association between a variation in calpain 10 (CAPN10) gene and the response to metformin treatment in patients with type 2 diabetes. Eur J Clin Pharmacol. 2015; 71(1): 59-63.

47. Chung J-Y, Cho SK, Kim TH, Kim KH, Jang GH, $\mathrm{Kim} \mathrm{CO}$, et al. Functional characterization of MATE2-K genetic variants and their effcts on metformin pharmacokinetics. Pharmacogenet Genomics. 2013; 23(7): 365-373.

48. Speidel JT, Xu M, Abdel-Rahman SZ. Promoter haplotypes of the ABCB1 gene encoding the p-glycoprotein differentially affect its promoter cctivity by altering transcription factor binding. DNA Cell Biol. 2018; 37(12): 973-981. 
